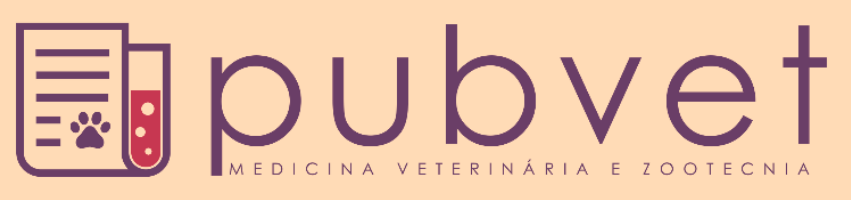

ISSN 1982-1263

https://doi.org/10.31533/pubvet.v13n9a421.1-5

\title{
Enterectomia no tratamento de carcinoide neuroendócrino obstrutivo em um cão: relato de caso
}

\author{
Patrícia Silva Vives $^{1^{*}}$, Marina $\operatorname{Zanin}^{2} \bullet$, Francisco de Assis Araújo Camelo Júnior ${ }^{3} \bullet$, Liliane \\ Cristina Dias Jerônimo ${ }^{4}$, Tainá Ança Evaristo ${ }^{5}{ }^{\circ}$, Márcia Kutscher Ripoll ${ }^{\circ} \bullet$, Stella Falkenberg \\ Rausch $^{7}{ }^{\circ}$, Michele Berselli ${ }^{8} \odot$, Cristina Gevehr Fernandes ${ }^{9}{ }^{\circ}$, Fabrício de Vargas Arigony Braga ${ }^{10} \odot$ \\ ${ }^{I}$ Médica Veterinária, doutora, Técnica em Educação no Hospital de Clínicas Veterinárias da Universidade Federal de Pelotas \\ (HCV-UFPel). Pelotas-RS, Brasil. \\ ${ }^{2}$ Médica Veterinária, pós-graduanda em Ciências Fisiológicas, Universidade Federal de Rio Grande, Rio Grande - RS, Br. \\ ${ }^{3}$ Médico Veterinário, UFPel, Pelotas-RS, Br. \\ ${ }^{4}$ Médica Veterinária, Residente em Clínica Cirúrgica de Animais de Companhia UFPel, Pelotas-RS, Br \\ ${ }^{5}$ Graduanda em Medicina Veterinária, UFPel, Pelotas- $R S, B r$. \\ ${ }^{6}$ Médica Veterinária, Doutoranda em Ciências Veterinária, área de Farmacologia, UFPel, Pelotas-RS, Br. \\ ${ }^{7}$ Médica Veterinária, Imagenologista na Clínica de Imagem Stella Falkenberg Rausch, UFPel, Pelotas-RS, Br. \\ ${ }^{8}$ Médica Veterinária, Doutoranda em Ciências Veterinária, área de Patologia Animal, UFPel, Pelotas-RS, Br \\ ${ }^{9}$ Docente do curso de Medicina Veterinária, Departamento de Patologia Animal, UFPel, Pelotas-RS, Br. \\ ${ }^{10}$ Docente do curso de Medicina Veterinária, Departamento de Clínicas Veterinárias, UFPel, Pelotas-RS, Br. \\ *Autor para correspondência, E-mail: patvivesvet@hotmail.com
}

Resumo. Carcinoides em cães são neoplasias raras do sistema celular neuroendócrino difuso. O termo tumor neuroendócrino tem sido proposto e a manifestação clínica está relacionada com a localização das massas. Apesar da baixa velocidade de crescimento e de metastatização, tais neoplasias frequentemente são malignas e devem ser submetidas a ressecção cirúrgica. Quando esta é realizada adequadamente, o desfecho geralmente é satisfatório. Objetivou-se descrever um caso de carcinoide em uma cadela de dez anos apresentando tenesmo e fecaloma. A paciente foi recebida em uma clínica veterinária particular e, após consulta clínica e exames de imagem, foi submetida à enterotomia para a remoção do fecaloma localizado em cólon descendente. A obstrução do fluxo intestinal decorreu de massa neoplásica em região pré-púbica, a qual foi resseccionada e encaminhada para análise. O histopatológico revelou carcinoide neuroendócrino e a cadela foi acompanhada por 60 dias pós procedimento, sem sinais de recidiva ou metástases. Diante disso, entende-se que carcinoides neuroendócrinos devem ser considerados como causa de tenesmo e constipação em cães, especialmente nos idosos, e o prognóstico pode ser favorável quando os pacientes livres de metástases são submetidos ao procedimento cirúrgico com ressecção adequada do tumor.

Palavras chave: constipação, fecaloma, tumor, carcinoma

\section{Enterectomy in the treatment of obstructive neuroendocrine carcinoid in a dog: case report}

Abstract. Carcinoids in dogs are rare neoplasms of the diffuse neuroendocrine cell system.
The term neuroendocrine tumor has been proposed and the clinical manifestation is related
to the location. Despite the low rate of growth and metastasis, such neoplasms are often
malignant and must undergo surgical resection. When this is done properly, the outcome is
usually satisfactory. The objective of this study was to describe a case of carcinoid in a 10-
year-old female with tenesmus and presence of fecaloma. The patient was received at a
private veterinary clinic and, after clinical consultation and imaging examinations, she was 
submitted to the enterotomy and removal of fecaloma in the descending colon. The obstruction of the intestinal flow was resulting from a neoplastic mass in a pre-pubic region, also resected and forwarded for analysis. The histopathological revealed neuroendocrine carcinoid and the dog was followed for 60 days after the procedure, without signs of recurred or metastasis. Therefore, it is understood that neuroendocrine carcinoids should be considered as a cause of tenesmus and constipation in dogs, especially in the elderly, and the prognosis is favorable when patients undergo the surgical procedure with adequate resection of the tumor.

Keywords: constipation, fecaloma, tumor, carcinoma

\section{Enterectomia en el tratamiento del carcinoide neuroendocrino obstructivo en un perro: relato de caso}

Resumen. Carcinoides en perros son neoplasias raras del sistema celular neuroendocrino difuso. El término tumor neuroendocrino ha sido propuesto y la manifestación clínica está relacionada con la localización. A pesar de la baja velocidad de crecimiento y de metástasis, tales neoplasias a menudo son malignas y deben ser sometidas a la resección quirúrgica. En cuanto se realiza adecuadamente, el resultado generalmente es satisfactorio. Se objetivó describir un caso de carcinoide en perra de diez años presentando tenesmo y presencia de fecaloma. La paciente fue recibida en una clínica veterinaria particular y, tras consulta clínica y exámenes de imagen, fue sometida a la remoción de fecaloma en colon descendente. La obstrucción del flujo intestinal transcurrió de masa neoplásica en región prepúica, la cual también fue reseccionada y encaminada a análisis. El histopatológico reveló carcinoide neuroendocrino y la perra fue acompañada por 60 días después del procedimiento, sin signos de recidiva o metástasis. Por lo tanto, se entiende que los carcinoides neuroendocrinos deben considerarse como causa de tenesmo y constipación en perros, especialmente en los ancianos, y el pronóstico es favorable cuando los pacientes son sometidos al procedimiento quirúrgico con resección adecuada del tumor.

Palabras clave: constipación, fecaloma, tumor, carcinoma

\section{Introdução}

Os tumores carcinoides são neoplasias do sistema neuroendócrino difuso e raramente identificados em cães e podem ter diferentes perfis de produção hormonal de acordo com o sítio de origem. Quando malignos, podem produzir peptídeos vasoativos e hormonais, gerando diversas reações orgânicas e originando diferentes quadros clínicos (Creutzfeldt, 1996; Niwa \& Nico, 2008; Raskin \& Meyer, 2011). A sintomatologia varia de acordo com a localização da massa, dificultando o diagnóstico a partir do quadro clínico, sendo rara a hipótese pré-operatória de carcinoide (Creutzfeldt, 1996). O diagnóstico ocorre por meio do exame clínico e físico do animal, exames de imagem e principalmente o histopatológico (Nagase et al., 2011). Embora os carcinoides sejam raros em cães, os gastrointestinais são os mais prevalentes. A exérese do tumor consiste na primeira escolha terapêutica, tanto com finalidade curativa como paliativa (Creutzfeldt, 1996; Niwa \& Nico, 2008; Raskin \& Meyer, 2011).

O presente trabalho objetiva descrever um caso de carcinoide neuroendócrino obstrutivo em cólon descendente, em uma cadela acometida por constipação e tenesmo, com excelente recuperação após exérese tumoral.

\section{Relato de caso}

Foi levada a uma clínica particular na cidade de Pelotas/RS, uma cadela sem raça definida, aproximadamente dez anos de idade e massa corpórea de sete quilos, com histórico de tenesmo e constipação. Ao exame clínico foi constatado a presença de massa abdominal firme em região de cólon descendente. Foram realizados exames de imagem, quando a radiografia abdominal (Figura 1) revelou a presença de um fecaloma que se estendia desde o cólon transverso até o púbis, terminando de forma abrupta. 

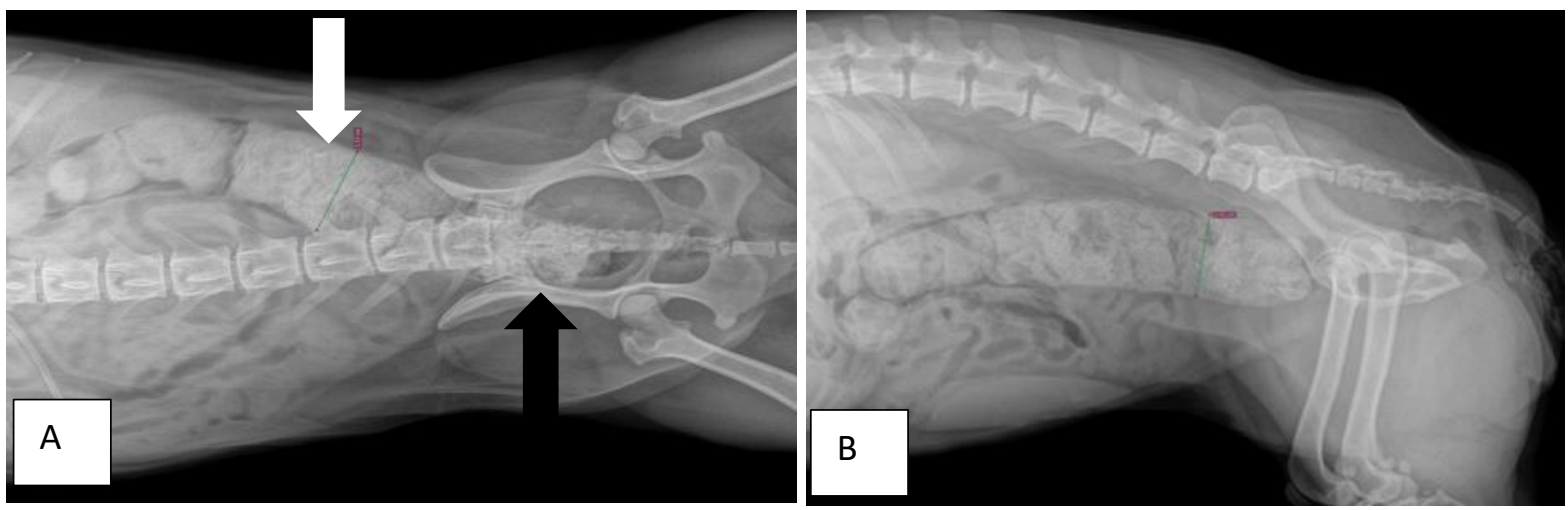

Figura 1. (A) Imagem radiográfica de uma cadela em posição ventro-dorsal revelando distensão do colón descendente repleto por fezes, formando um fecaloma (seta branca) e ausência de conteúdo fecal na ampola retal em região onde a massa estava localizada, produzindo a obstrução do fluxo fecal (seta preta). (B) Imagem radiográfica latero- lateral demonstrando diâmetro do fecaloma.

Após estabilização e preparo prévio, a paciente foi encaminhada ao setor cirúrgico objetivando realizar o procedimento de enterotomia. Entretanto, à celiotomia verificou-se duas massas além do fecaloma, uma posicionada cranial ao púbis (Figura 2A), extramural e discretamente aderida à serosa do cólon descendente e ilíacas esquerdas interna e externa (Figura 2B) de aproximadamente 6 x $4 \mathrm{~cm}$ (Figura 2C), e outra cranial a esta com aderência no omento maior de $1 \times 2 \mathrm{~cm}$.

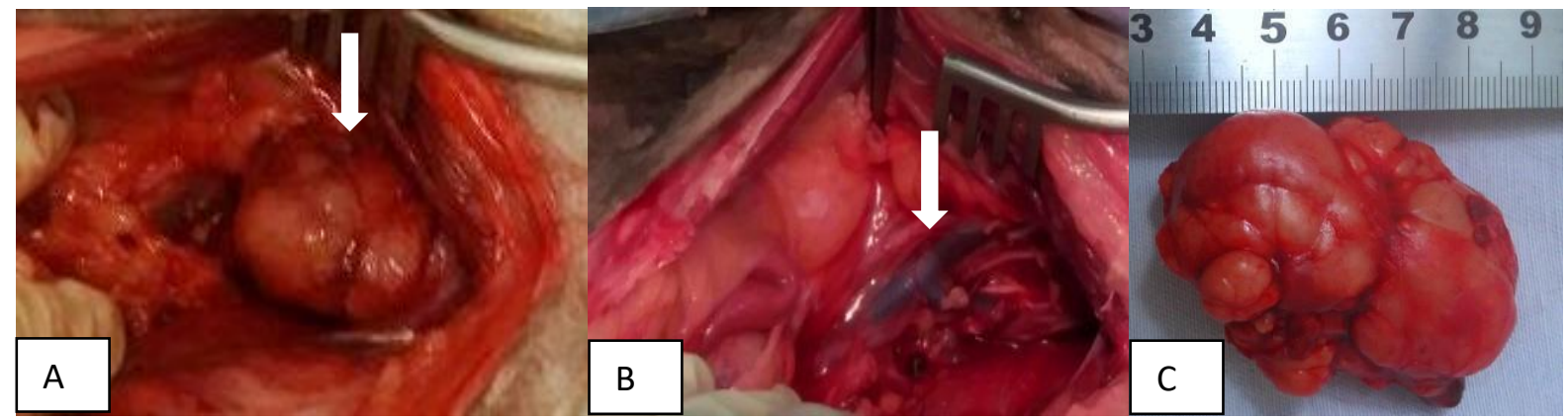

Figura 2. (A) Massa tumoral evidenciada no transcirúrgico de uma cadela, localizada na cavidade abdominal cranial ao púbis, obliterando parcialmente o canal pélvico de uma cadela (seta). (B) Cavidade abdominal após a ressecção de massa tumoral, evidenciando o local de aderência de uma massa tumoral sob a bifurcação da veia ilíaca (seta). (C) Massa tumoral de aproximadamente 6 x 4 cm, após ressecção da cavidade abdominal de uma cadela.

Após enterectomia de um segmento do colón descendente comprometido pela massa, remoção do conteúdo fecal e enterorrafia de rotina, procedeu-se a irrigação copiosa da cavidade abdominal com solução salina $0,9 \%$ aquecida e substituição da paramentação e do material para executar a exérese destes tumores por meio de divulsão com auxílio da tesoura romba delicada e ligadura dos vasos. Na sequência, fez-se celiorrafia de rotina e a paciente foi mantida hospitalizada por 24 horas, tendo alta com prescrição de medicação analgésica e antimicrobiana.

As massas foram enviadas para análise histopatológica e eram constituídas por cordões ou ninhos de células de tamanho médio, redondas a ovaladas, com citoplasma eosinofílico moderado e, por vezes, fibrilar (Figura 3A). Os núcleos eram arredondados e hipercromáticos e numerosas mitoses, pleomorfismo moderado, presença de rosetas e pseudo-rosetas foram observadas (Figura 3B). Grupos celulares delimitados por estroma fibrovascular delicado e pequenos focos de necrose.

O diagnóstico foi firmado em carcinoide (carcinoma neuroendócrino bem diferenciado). A cadela foi acompanhada por 60 dias de pós-operatório e apresentava-se bem, sem sinais de recidiva do neoplasma.

\section{Discussão}

A bibliografia veterinária acerca de tumores neuroendócrinos em cães é escassa, porém, trabalhos citam maior prevalência em animais acima de sete anos, conforme verificado no presente relato. Com 
relação à predileção por raça ou sexo, embora não haja citação, estudos em humanos demonstram maior acometimento de indivíduos do sexo feminino (Nia \& Nico, 2008; Raskin \& Meyer, 2011).

Os sinais clínicos normalmente inespecíficos estão diretamente relacionados a localização anatômica do tumor e quando acometem o intestino, os pacientes podem apresentar hemorragia intestinal e/ou vômito, anorexia, perda de peso, diarreia, desidratação e anemia (Nagase et al., 2011; Raskin \& Meyer, 2011). Apesar disso, a sintomatologia apresentada pela paciente corrobora a literatura de Bonagura \& Kirk (1992), a qual assemelha a sintomatologia a outros processos obstrutivos, como intussuscepção e corpo estranho, uma vez que as massas estavam extramurais. Os sinais de constipação e tenesmo justificavam-se pela localização do tumor em cólon descendente, e pelo grande volume em proporção ao porte do animal, foi capaz de obliterar parcialmente o canal pélvico.

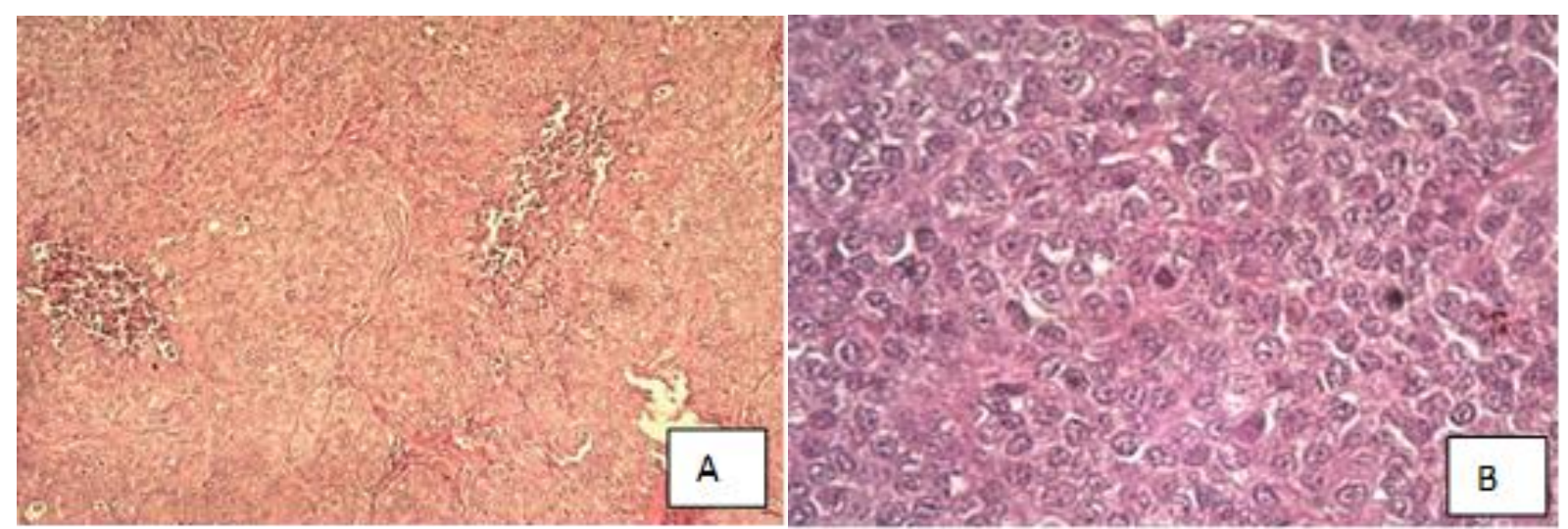

Figura 3. Imagens histopatológicas $\mathrm{HE}$ em aumento $10 \mathrm{x}$ revelaram células constituídas por cordões ou ninhos de tamanho médio, redondas a ovaladas, com citoplasma eosinofílico moderado e, por vezes, fibrilar (A). Em HE aumento 40 x, os núcleos eram arredondados e hipercromáticos apresentando numerosas mitoses, pleomorfismo moderado com presença de rosetas e pseudo-rosetas (B).

Em relatos de humanos acometidos por carcinoides extra intestinal foi possível verificar a Síndrome Carcinoide, causada pelos mediadores neuroendócrinos secretados pelo tumor, caracterizada por Cushing, diarreia, dor abdominal, broncoespasmo e doença valvar cardíaca (Niwa \& Nico, 2008). Apesar disso, Klöppel et al. (1996) não relaciona qualquer síndrome descrita associada a tumores neuroendócrinos em cães.

No presente relato o tutor não referiu sinais endócrinos, provavelmente por se tratar de um subtipo não secretor ou, ainda, embora as células dos carcinoides possam apresentar grânulos contendo diversas aminas bioativas, não há relatos em animais de que estas substâncias possam desencadear sintomatologia. Além disso, a etiopatogenia dos carcinoides em cães não está elucidada, tendo em vista o reduzido número de relatos.

Exames de imagem são úteis na suspeita de tumores intestinais, contudo, o diagnóstico definitivo de tumor neuroendócrino só é obtido após o exame histopatológico mediante a remoção cirúrgica da massa (Creutzfeldt, 1996; Nagase et al., 2011). Naqueles em que há a liberação de substâncias, o diagnóstico pode ser sugestivo por meio da mensuração do 5-hidroxiindolacético na urina, um metabólito oriundo da serotonina (Raskin \& Meyer, 2011). Na maioria dos casos, o diagnóstico é obtido no pós-operatório por meio histopatológico, e apesar da dificuldade em predizer o comportamento biológico destes carcinoides com base na morfologia celular, este exame possibilita a classificação em tumores neuroendócrinos bem diferenciados e pouco diferenciados (Raskin \& Meyer, 2011).

A exérese desta neoplasia foi realizada após a enterotomia, uma vez que o fecaloma ocupava boa parte do abdômen, dificultando a identificação e a ressecção das massas. A remoção cirúrgica consiste na primeira escolha terapêutica, tanto com finalidade curativa como paliativa e quando são removidos por completo e as metástases estão ausentes, o prognóstico é favorável (Niwa \& Nico, 2008).

Após o procedimento cirúrgico, a paciente apresentou evolução satisfatória e manteve-se livre da doença no período de seguimento, corroborando o descrito por Mangueira et al. (2006). 


\section{Conclusão}

Carcinoides neuroendócrinos devem ser considerados como causa de tenesmo e constipação em cães, especialmente nos idosos. O prognóstico é favorável quando os pacientes livres de metástases são submetidos ao procedimento cirúrgico com ressecção adequada do tumor.

\section{Referências bibliográficas}

Bonagura, J. D. \& Kirk, R. W. (1992). Current veterinary therapy: small animal practice (Vol. 13). Philadelphia, USA: WB Saunders.

Creutzfeldt, W. (1996). Carcinoid tumors: development of our knowledge. World Journal of Surgery, 20(2):126-131.

Klöppel, G., Heitz, P. U., Capella, C. \& Solcia, E. (1996). Pathology and nomenclature of human gastrointestinal neuroendocrine (carcinoid) tumors and related lesions. World Journal of Surgery, 20(2):132-141.

Mangueira, P. A., Fernandes, G. O., Primo, C. C., França, M. A. V., Maia, H. P. \& Costa, J. H. G. (2006). Tumor carcinóide de reto. Revista Brasileira de Coloproctologia, 26(2):162-167.

Nagase, N. F., Coutinho, A. S., Bittencourt, G. C., Coelho, V. S., Fiuza, B., M,, Prada, B., M. \& Kolber, M. (2011). Neoplasia de intestino delgado de cães: Relato de caso. Revista de Educação Continuada em Medicina Veterinária e Zootecnia do CRMV-SP, 9(2):42-43.

Niwa, A. B. M. \& Nico, M. M. S. (2008). Síndrome carcinóide-Relato de caso. Anais Brasileiros de Dermatologia, 83(6):549-553.

Raskin, R. E. \& Meyer, D. J. (2011). Citologia de Cães e Gatos: atlas colorido e guia de interpretação. Rio de Janeiro, Brasil: Elsevier Brasil.

Recebido: 8 de julho, 2019.

Aprovado: 2 de agosto, 2019.

Publicado: 9 de outubro, 2019.

Licenciamento: Este artigo é publicado na modalidade Acesso Aberto sob a licença Creative Commons Atribuição 4.0 (CC-BY 4.0), a qual permite uso irrestrito, distribuição, reprodução em qualquer meio, desde que o autor e a fonte sejam devidamente creditados. 